Supporting Information:

\title{
Probing Liquid-Liquid Phase Separation in Secondary Organic Aerosol Mimicking Solutions Using Articulated Straws
}

Emmaline R. Longnecker, ${ }^{1}$ Lucy Metz, Rebecca S. Miller, ${ }^{2}$ Andrew E. Berke*

Department of Chemistry, Smith College, Northampton, MA 01063

* Corresponding author: Andrew E. Berke (aberke@smith.edu)

${ }^{1}$ Present address: Department of Chemistry, University of Colorado-Boulder, Boulder, CO 80309

2 Present address: illumis Inc., New York, NY 10013 


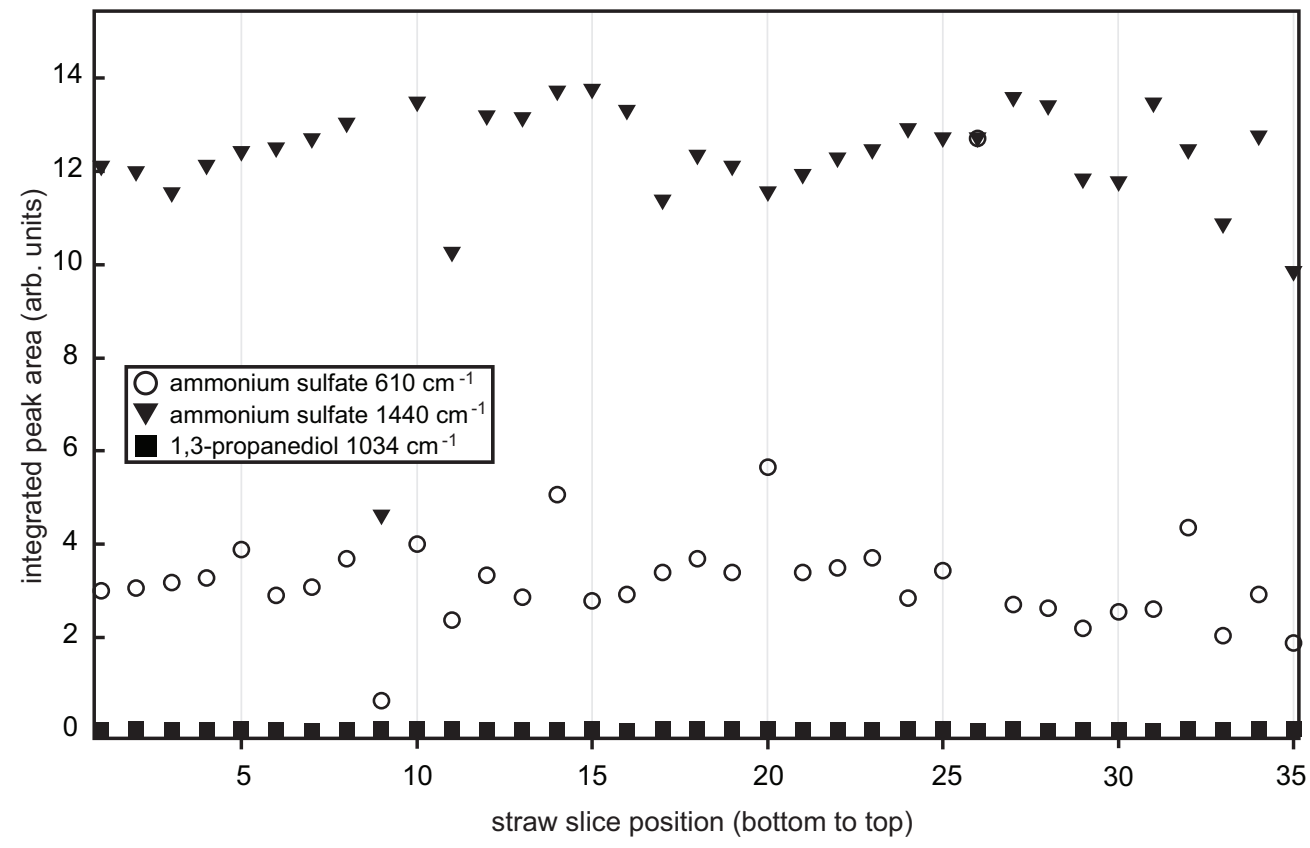

Figure S1. Integrated peak areas for solutions of 1,3-propanediol and ammonium sulfate as a function of solution depth (with slice 1 corresponding to the bottom of the straw) for two different ammonium sulfate peaks.

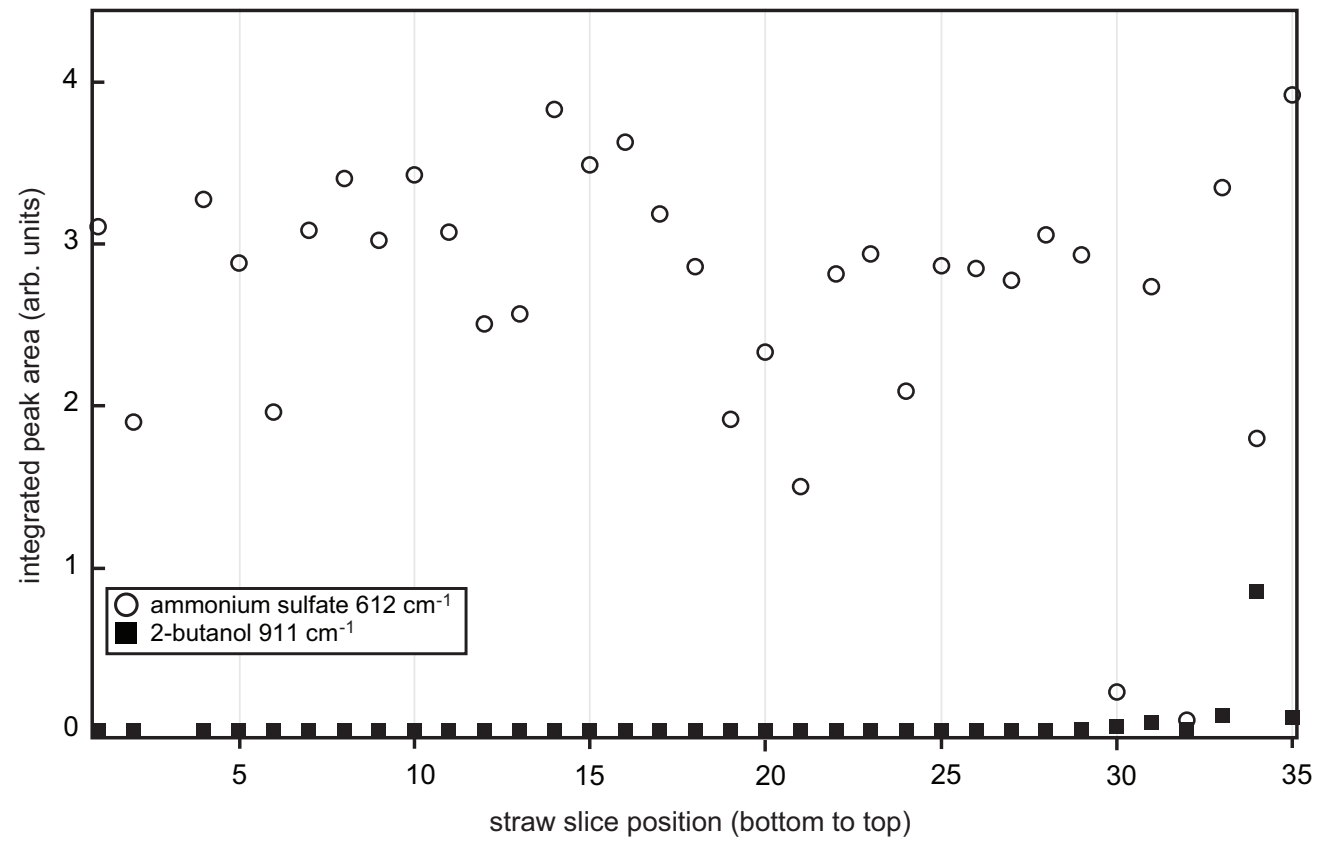


Figure S2. Integrated peak areas for solutions of 2-butanol and ammonium sulfate as a function of solution depth (with slice 1 corresponding to the bottom of the straw).

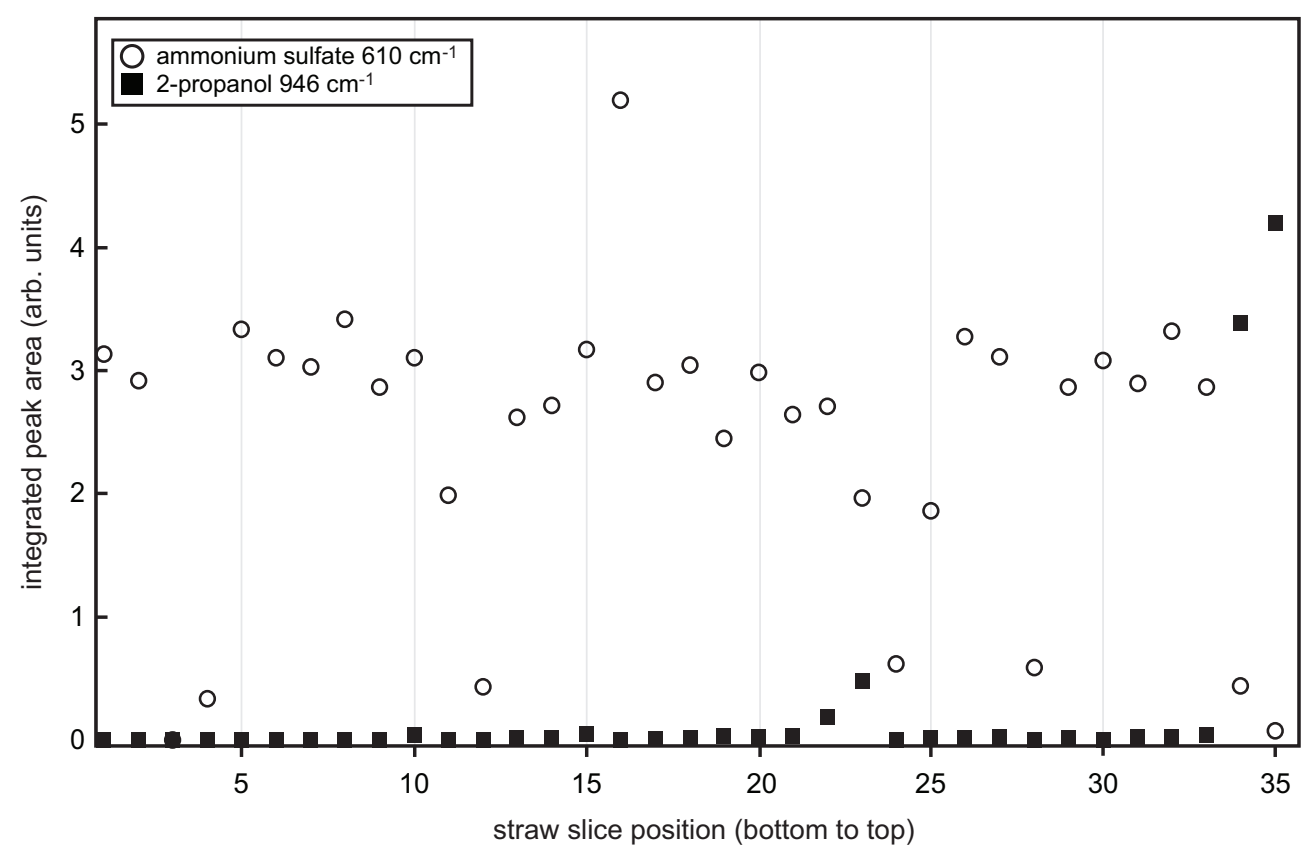

Figure S3. Integrated peak areas for solutions of 2-propanol and ammonium sulfate as a function of solution depth (with slice 1 corresponding to the bottom of the straw). 


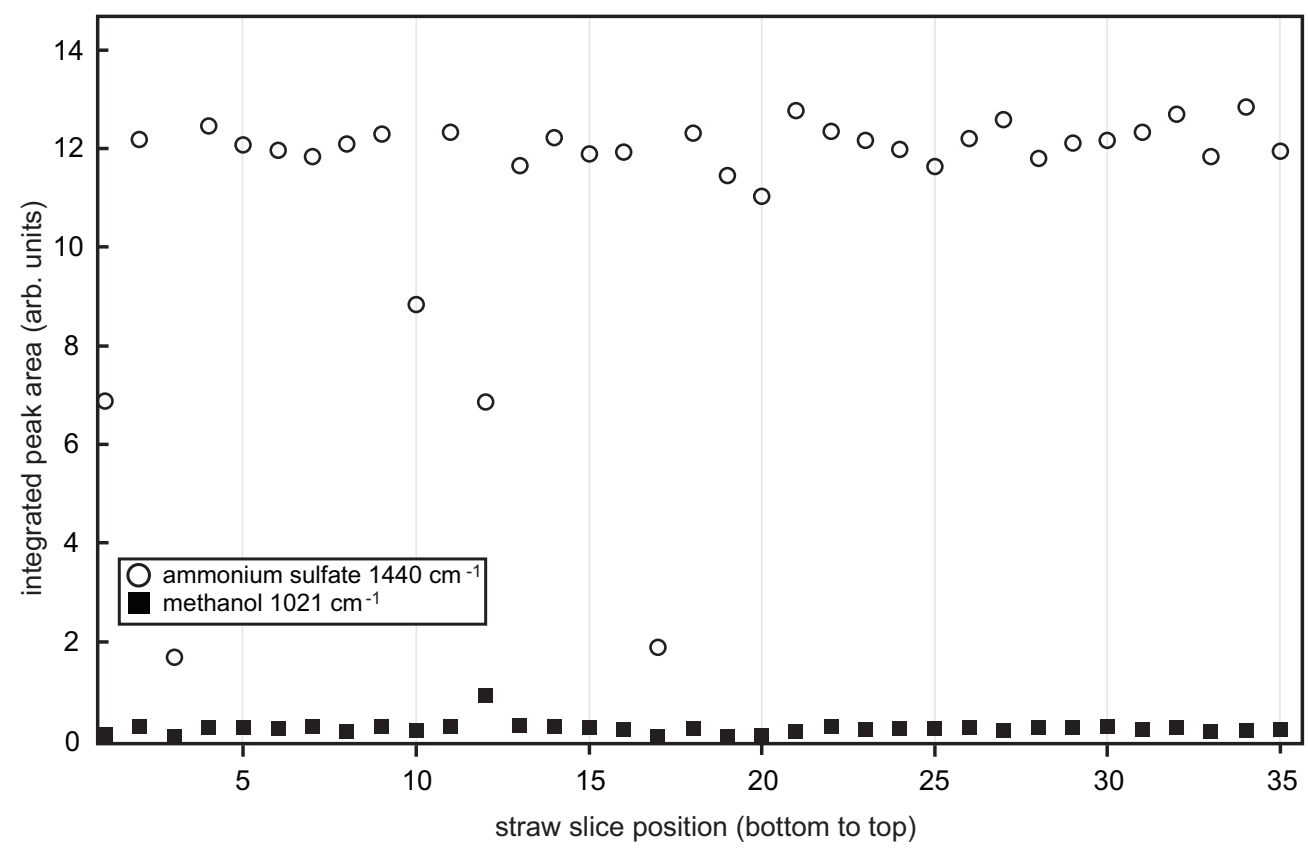

Figure S4. Integrated peak areas for solutions of methanol and ammonium sulfate as a function of solution depth (with slice 1 corresponding to the bottom of the straw).

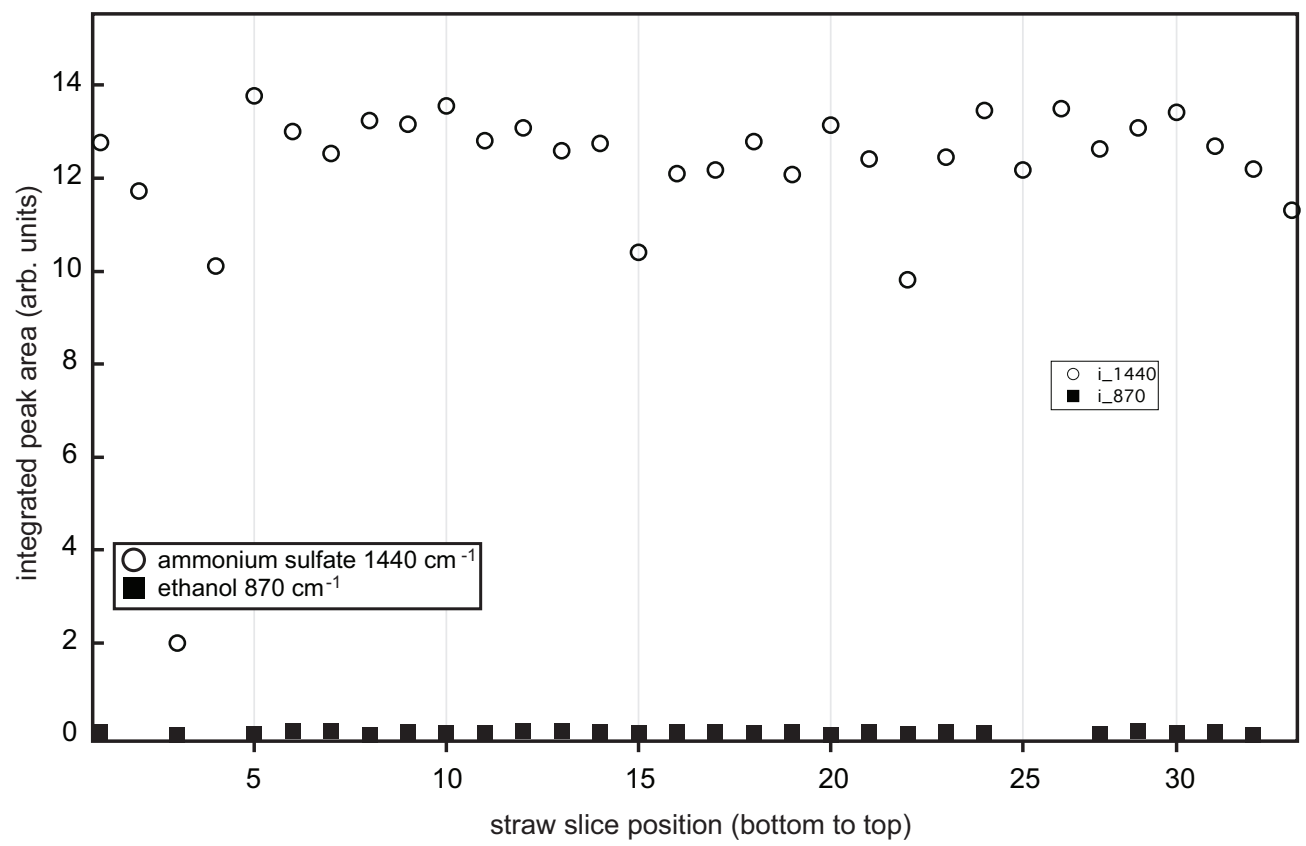

Figure S5. Integrated peak areas for solutions of ethanol and ammonium sulfate as a function of solution depth (with slice 1 corresponding to the bottom of the straw). 


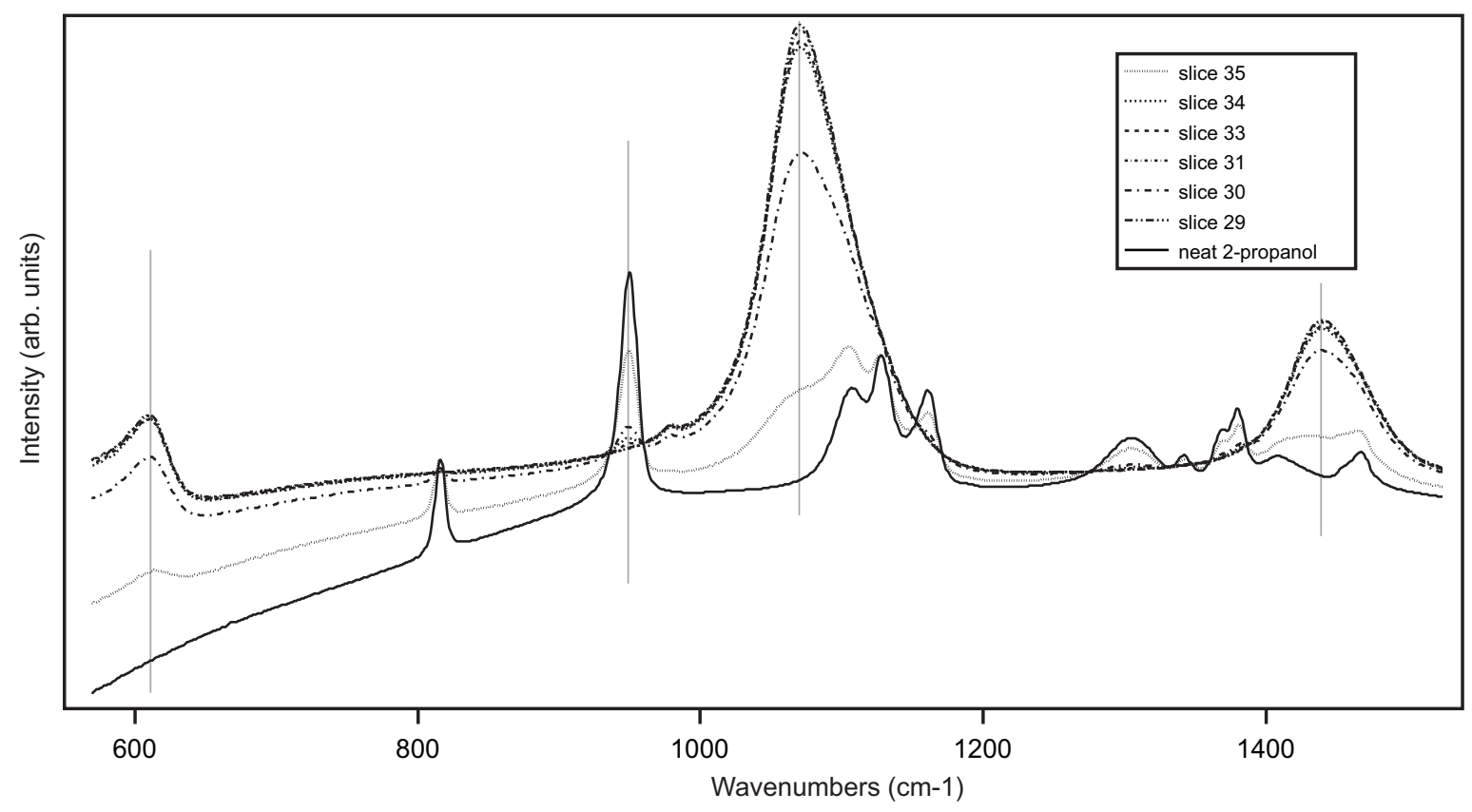

Figure S6. IR spectra for different depths in an articulated straw for 3.0 M ammonium sulfate solution containing 1.0 M 2-propanol. Spectra were processed using a water background. Vertical lines are to guide the eye and show the location of several alcohol and ammonium sulfate peaks.

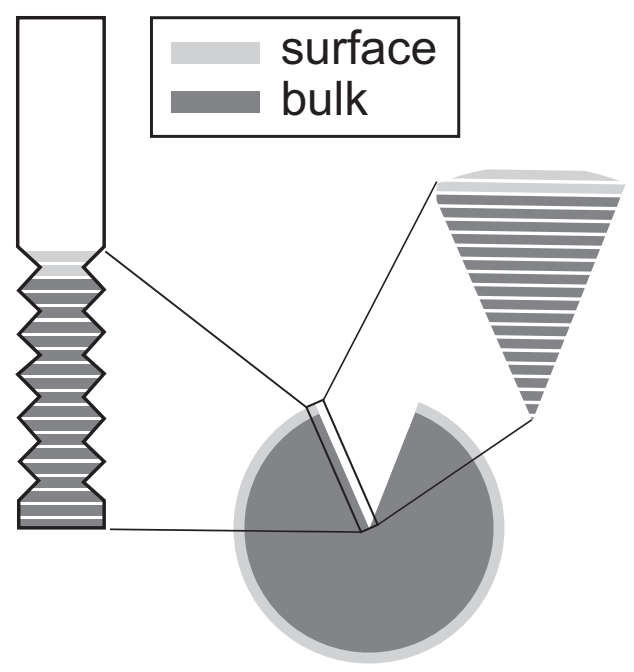

Figure S7. Schematic of the anticipated relationship between the articulated straw slices and a spherical aerosol particle. 


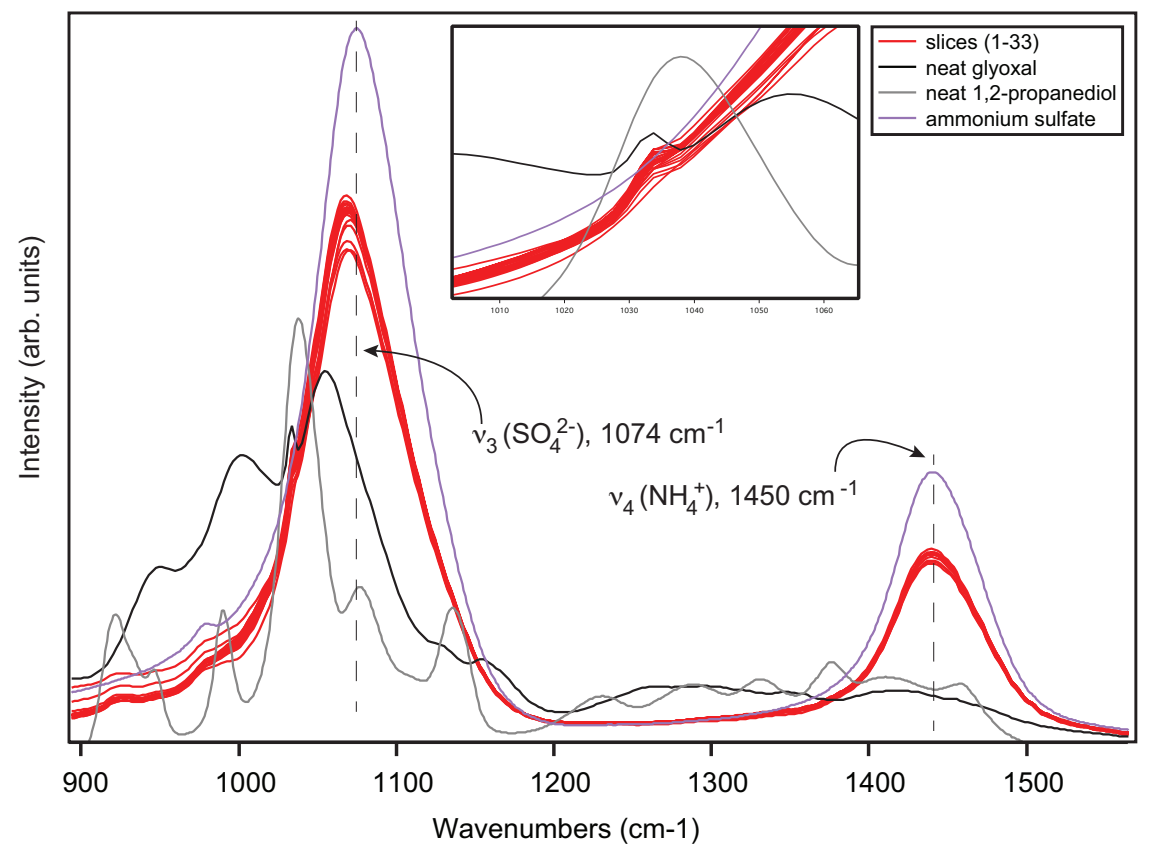

Figure S8. IR spectra for different depths in an articulated straw for 3.0 M ammonium sulfate solutions containing 1,2-propanediol and glyoxal. The inset is a close up of the glyoxal peak used for analysis, at $1033 \mathrm{~cm}^{-1}$. 